\title{
On the design of a triaxial accelerometer
}

\author{
Joost C Lötters, Wouter Olthuis, Peter H Veltink and Piet \\ Bergveld
}

MESA Research Institute, University of Twente, PO Box 217, 7500 AE Enschede, The Netherlands

Received 14 December 1994, accepted for publication 4 January 1995

\begin{abstract}
Up to now, mainly uniaxial accelerometers are described in most publications concerning this subject. However, triaxial accelerometers are needed in the biomedical field. Commercially available triaxial accelerometers consisting of three orthogonally positioned uniaxial devices do not meet all specifications of the biomedical application. Therefore, a new highly symmetrical inherently triaxial accelerometer is being developed, the advantages of which are higher sensitivity and reduction of off-axis sensitivity.
\end{abstract}

\section{Introduction}

Accelerometers are being used in shock, movement and vibration measurements, for instance in airbag systems. Since the mass of the device is not allowed to influence the measurement results, miniaturization is demanded. Therefore, it is necessary to design and to realize reliable micromechanical accelerometers.

Accelerometers are also being used in the biomedical field, including by the Biomedical Engineering Division of the University of Twente [1]. The research done on the accelerometer as described in this paper will contribute to the development of a functional electrical stimulation (FES) system which will eventually enable paraplegic patients to walk on their own again. In this application, the devices must be small (in vivo use), stable and preferably selfcalibrating. Moreover, the devices must be biocompatible and should have low power consumption (battery lifetime).

Specifically, the devices need to be sensitive in three directions (triaxial) in the range between $-5 g$ and $+5 g$ (with $g$ being the earth's gravitational force). Also, a frequency response from 0 to $50 \mathrm{~Hz}$ and a dimension of $2 \times 2 \times 2 \mathrm{~mm}^{3}$ are required.

Up to now, mainly uniaxial accelerometers have been discussed in most publications concerning this subject [24]. The main principle described is the mass-spring-damper system. When the mass is subjected to an acceleration, the length of the spring is changed. The main methods to detect this change in length are the capacitive method, the piezoresistive method and the piezoelectric method. The capacitive method is the most sensitive and the least power consuming.

Few inherently triaxial accelerometers have been presented $[5,6]$. Inherently triaxial means that the device does not consist of three uniaxial sensors, but that it is truly triaxial. A disadvantage of the presented triaxial devices is that the off-axis sensitivity is rather high because of the lack of symmetry. Therefore, when a highly symmetrical design is made, this problem can be reduced.

The purpose of this research is to develop a prototype of the triaxial accelerometer. The structure of the device is a central mass surrounded by capacitors. The change of the capacitance in any direction, as caused by the enclosed central mass, is a measure of the occurring aceéleration. Present research is focused on the realization.' 'of this accelerometer structure.

\section{Present sensors}

Commercially available triaxial accelerometers consist of three orthogonally positioned uniaxial devices with one of the conventional mass-spring configurations [2-4]. A disadvantage of this construction is that the sensitivity is not necessarily equal in all directions, making an individual calibration procedure necessary for each axis. In addition, the device is at least three times as big as in the uniaxial case. Unfortunately, it is not possible to extend one of the uniaxial mass-spring structures to an inherently triaxial accelerometer. A new structure is being developed and some alternatives are discussed in the next section.

\section{Alternatives}

\subsection{Deformable mass}

For the new generation of acceleration and position sensors, the already developed principle of the applanation sensor which is part of the eyeball pressure sensor [7] seemed to be interesting. The applanation sensor is shown in figure 1. When the sensor is used to flatten the eye globe, the foil is pressed against the metal contacts, resulting in a short cut 


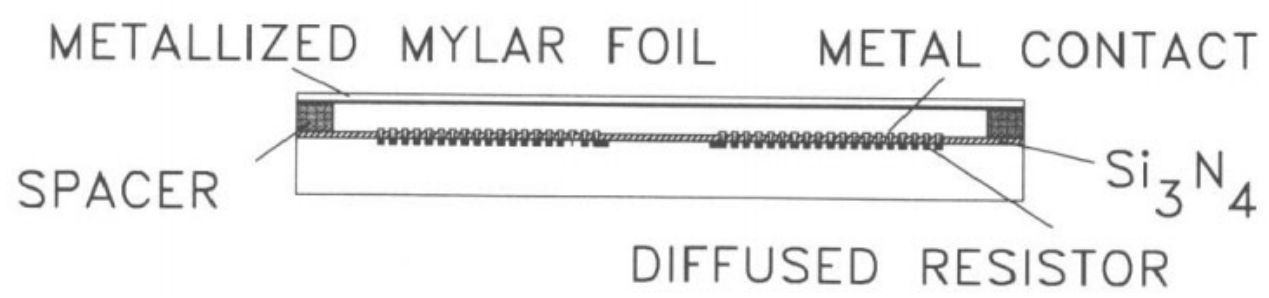

Figure 1. The applanation sensor [7].

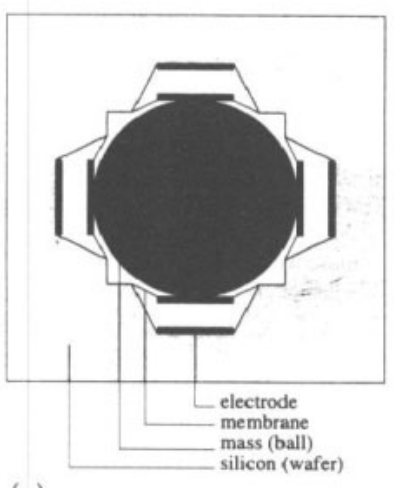

(a)

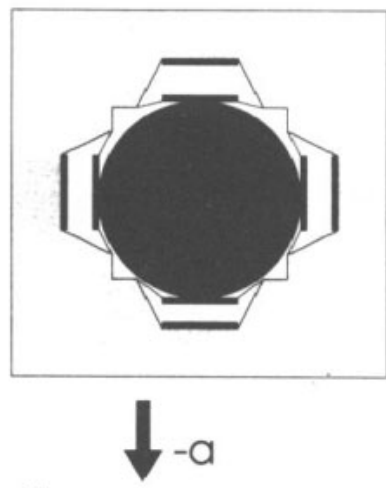

(b)

Figure 2. Schematic cross-sectional view of the inherently triaxial accelerometer; (a) at rest; (b) accelerated.

of parts of the resistors. The radius of the flattened area can be calculated from the values of the four resistors.

The applanation sensors can be mounted in a cubic arrangement by folding identical sensors into a cube. A compliant mass should be fitted inside the cube. Due to acceleration of the assembly, including gravitation, the shape of this mass will be changed which is detected by the applanation sensors.

The development of a suitable mass was part of this research project. The right choice of the materials and geometry should have led to the desired bandwidth, measurement range, low hysteresis and low drift. Unfortunately, it was recently shown that no material so far has the properties necessary to fulfil the above mentioned demands. Therefore, this principle will not be useful in this application.

\subsection{Undeformable mass}

The new triaxial accelerometer design can consist of capacitors (with a rigid backplate at the one side and a deformable membrane at the other) with a mass enclosed in between, as shown in figure 2(a). When an acceleration is applied, the mass will deform the membranes (which can be seen as springs) of the capacitors, as is shown in figure $2(b)$. The change in capacitance due to the deformation of the membranes can be detected and is a measure for the applied acceleration. The air between the membrane and the backplate of the capacitor causes damping with which the suitable frequency range can be tuned [8].

Research will concentrate on the feasibility of this design, first only tested in the uniaxial mode. Silicon miniature capacitors (microphones from an existing bearing

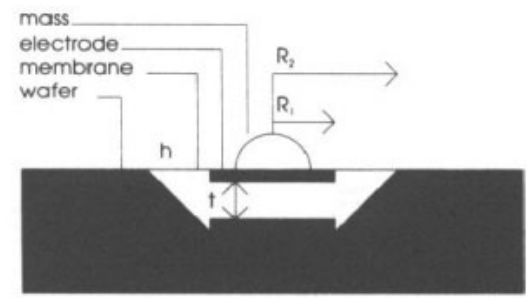

Figure 3. A silicon based capacitor with a mass attached to the membrane.

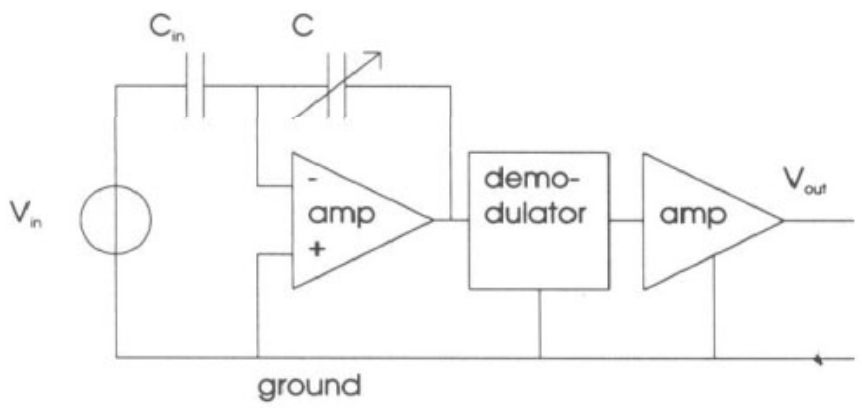

Figure 4. Schematic drawing of the capacitance to voltage converter.

aid project [9]) can be used to obtain some experimental results.

\section{Theory}

The centre deflection $w(0) \mathrm{m}$ of a membrane with a mass attached to it, as shown in figure 3 , can be calculated with [10]

$$
w(0)=\frac{\left(\frac{1}{2}+\ln \left(R_{2} / R_{1}\right)\right) m a}{2 \pi \sigma h}
$$

with $R_{1}$ radius of the mass, $9.10 * 10^{-4} \mathrm{~m}, R_{2}$ radius of the membrane, $1.38 \times 10^{-3} \mathrm{~m}, m$ mass, $7.9 \times 10^{-6} \mathrm{~kg}, a$ applied acceleration $\mathrm{m} \mathrm{s}^{-2}, \sigma$ initial stress in the membrane, $7.3 \times 10^{6} \mathrm{~Pa}, h$ thickness of the membrane, $1.5 \times 10^{-6} \mathrm{~m}$.

When $w(0)$ is smaller than $t / 15$ the capacitance $C(\mathrm{~F})$ can be obtained using [11]

$$
C=\frac{\epsilon_{0} A}{t-\frac{1}{4} w(0)}
$$

with $\epsilon_{0}$ dielectric permittivity of vacuum, $8.85 \times 10^{-12} \mathrm{~F}$ $\mathrm{m}^{-1}, A$ area of the (electrode of) the membrane, $3.0 \times 10^{-6}$ $\mathrm{m}^{2}, t$ airgap between the membrane and the backplate at rest, $3.20 \times 10^{-6} \mathrm{~m}$. 


\section{MASS/MIC --- ICSensors}

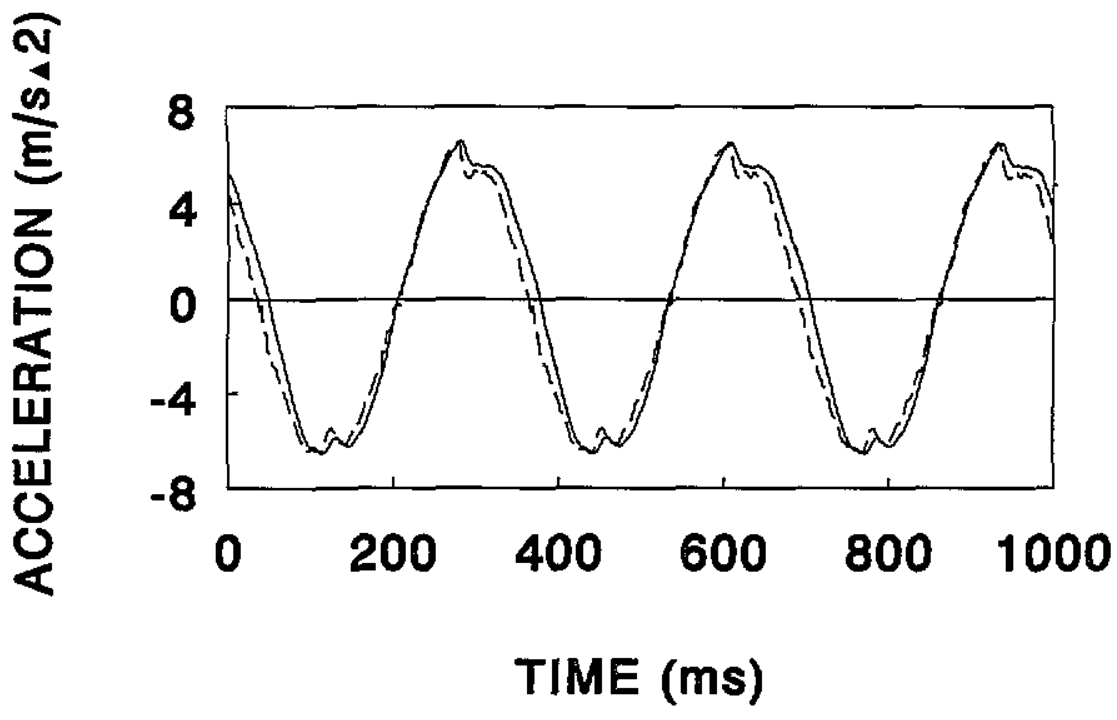

Figure 5. Measured acceleration of the mass/microphone and the ICSensors 3021.

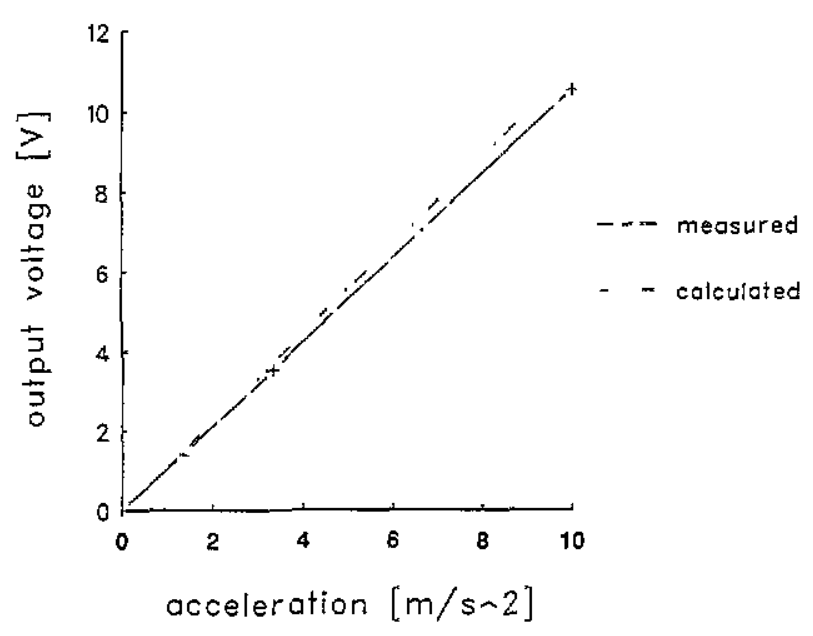

Figure 6. Measured and calculated output voltage versus the applied acceleration.

After conversion of the capacitance to a voltage (see figure 4) the output voltage $V_{\text {out }}[\mathrm{V}]$ is

$$
V_{\text {out }}=\frac{\frac{1}{8} w(0) C_{\text {in }} V_{\text {in }} A_{\text {vv }}}{\epsilon_{0} A}
$$

with $V_{\text {in }}$ applied input voltage, amplitude $4 \mathrm{~V}$ (frequency $50 \mathrm{kHz}$, sine wave), $A_{\mathrm{v}}$ amplification factor of the amplifier, $56, C_{\text {in }}$ input capacitor, $10 \mathrm{pF}$.

When all values are substituted in equation (3), it can be rewritten as

$$
a=0.90 V_{\text {out }}
$$

so, when the output voltage is known the acceleration can be calculated (figure 6 ).

\section{Experimental procedure}

\subsection{Device preparation}

The processing and fabrication of the silicon based capacitor is described in detail elsewhere [9]. The sensitive membrane of the capacitor consists of a gold plated $2.45 \times 2.45 \mathrm{~mm}^{2}$ Mylar foil which thickness is $1.5 \mu \mathrm{m}$. A spherical piece of chrome with a radius of $0.91 \mathrm{~mm}$ and a mass of 7.9 milligrams is glued on top of this membrane. This mass/capacitor should act like a uniaxial accelerometer.

\subsection{Measurement set-up}

Both the mass/capacitor and a commercially available uniaxial accelerometer (ICSensors 3021, which is used as reference) are mounted (with double-sided adhesive tape) on top of a shaker unit (including a linear displacement motor), their sensitive axes in the same direction. The mass/capacitor is connected to a capacitance-to-voltage converter (see figure 4). A computer collects the output voltages of both accelerometers and converts them into absolute accelerations.

\subsection{Measurement protocol}

Sine wave accelerations with the following amplitudes and frequencies are supplied:

-constant amplitude of $1 \mathrm{~g}$ : frequencies: $1,2,3,5,10$, $20,30,40,50 \mathrm{~Hz}$

-constant frequency of $50 \mathrm{~Hz}$ : amplitudes: $0.13 \mathrm{~g}$, $0.33 g, 0.66 g, 1 g$.

\section{Results and discussion}

A typical example of a measured acceleration curve is shown in figure 5 . It shows a sine wave acceleration in 
one direction with an amplitude of $0.66 \mathrm{~g}$ and a frequency of $3 \mathrm{~Hz}$. The phase difference in the negative slope is caused by the damping which occurs when the airgap is decreased.

The measured acceleration amplitudes (corrected for the influence of parasitic capacitances) of the constant frequency of $50 \mathrm{~Hz}$ are shown in figure 6 . The figures 5 and 6 as well as the results of the other experiments show that the results of the mass/capacitor configuration correspond well to the results predicted by the theoretical model. The deviation between the measurements and the model (as shown in figure 6) is caused by the fact that equation (2) is only valid for small deflections (and therefore small accelerations).

The measurements show that this principle is suitable for the measuring of uniaxial accelerations and will therefore be useful in the inherently triaxial configuration.

\section{Conclusions}

Up to now, most papers on accelerometers treat uniaxial devices. However, in the functional electrical stimulation (FES) application for mobility restoration for paraplegics a small triaxial accelerometer is necessary. Therefore, a new inherently, triaxial prototype is being developed.

A triaxial accelerometer based on the idea of one central mass surrounded by capacitors is advantageous, since the sensitivity is higher and off-axis sensitivity is reduced. The capacitive detection method is the most sensitive and the least power consuming.

A uniaxial mass/capacitor configuration showed promising first results.

\section{Acknowledgment}

The authors would like to thank the Dutch Foundation for Applied Sciences (STW) for its financial support.

\section{References}

[1] Willemsen A Th M 1990 Accelerometers for gait assessment in functional neuromuscular stimulation $P h D$ Thesis University of Twente

[2] Roylance L M and Angell J B 1979 A batch-fabricated silicon accelerometer IEEE Trans. Electron Devices ED-26 27-33

[3] Chen P L, Muller R S and Andrews A P 1984 Integrated silicon PI-FET accelerometer with proof mass Sensors Actuators 5 119-26

[4] Crazzolara H, Flach G and von Munch W 1993 Piezoresistive accelerometer with overload protection and low cross-sensitivity Sensors Actuators A 39 201-7

[5] Jono K, Hashimoto M and Esashi M 1994 Electrostatic servo system for multi-axis accelerometers Proc. MEMS pp 251-6

[6] Okada K 1992 Tri-axial piezoresistive accelerometer Technical Digest 11th Sensor Symp. pp 245-8

[7] den Besten C 1993 Sensor systems for the measurement of intraocular pressure $P h D$ Thesis University of Twente

[8] Scheeper P R 1993 A silicon condenser microphone: materials and technology $P h D$ Thesis University of Twente

[9] van der Donk A G H 1992 A silicon condenser microphone: modelling and electronic circuitry $P h D$ Thesis University of Twente

[10] Morse P M and Ingard K U 1969 Theoretical Acoustics (New Yotk: McGraw-Hill)

[11] Puers R 1993 Capacitive sensors: when and how to use them Sensors Actuators A 37-38 93-105 